\title{
Gene expression analysis of vascular pathophysiology related to anti-TNF treatment in rheumatoid arthritis
}

Szilárd Póliska ${ }^{1,5+}{ }^{2}$, Timea Besenyei ${ }^{2,3 \dagger}$, Edit Végh², Attila Hamar², Anita Pusztai ${ }^{2}$, Andrea Váncsa², Nóra Bodnár ${ }^{2}$, Szilvia Szamosi ${ }^{2}$, Mária Csumita ${ }^{1,5}$, György Kerekes ${ }^{4}$, Zoltán Szabó2 ${ }^{2}$ Zoltán Nagy², Gabriella Szűcs², Sándor Szántó ${ }^{2,5}$, Gábor Zahuczky ${ }^{6}$, László Nagy ${ }^{1}$ and Zoltán Szekanecz ${ }^{2 *}$

\begin{abstract}
Objectives: Impaired vascular pathophysiology and increased cardiovascular (CV) mortality are associated with rheumatoid arthritis (RA). To date, no genomic analysis of RA- and RA treatment-related vascular pathophysiology has been published. In this pilot study, we performed gene expression profiling in association with vascular pathophysiology in RA patients.

Methods: Sixteen and 19 biologic-naïve RA patients were included in study 1 and study 2, respectively. In study 1 , genetic signatures determined by microarray were related to flow-mediated vasodilation (FMD), pulse-wave velocity (PWV), and common carotid intima-media thickness (IMT) of patients. In study 2, clinical response (CR) vs non-response (CNR) to 1-year etanercept (ETN) or certolizumab pegol (CZP) treatment, as well as "vascular" response (vR) vs non-response (vNR) to biologics, were also associated with genomic profiles. Multiple testing could not be performed due to the relatively small number of patients; therefore, our pilot study may lack power.

Results: In study 1, multiple genes were up- or downregulated in patients with abnormal vs normal FMD, IMT, and PWV. In study 2, there were $13 \mathrm{CR}$ and $6 \mathrm{cNR}$ anti-tumor necrosis factor (TNF)-treated patients. In addition, 10, 9, and 8 patients were FMD-20\%, IMT-20\%, and PWV-20\% responders. Again, vascular responder status was associated with changes of the expression of various genes. The highest number of genes showing significant enrichment were involved in positive regulation of immune effector process, regulation of glucose transport, and Golgi vesicle budding.

Conclusion: Differential expression of multiple genetic profiles may be associated with vascular pathophysiology associated with RA. Moreover, distinct genetic signatures may also be associated with clinical and vascular responses to 1-year anti-TNF treatment.
\end{abstract}

Keywords: Rheumatoid arthritis, Etanercept, Certolizumab pegol, Gene expression, Genetic signature, Atherosclerosis, Vascular pathology, Prediction, Response

\footnotetext{
* Correspondence: szekanecz.zoltan@med.unideb.hu; http://www. rheumatology.hu

Szilárd Póliska and Timea Besenyei are shared first authorship with equal contribution.

${ }^{2}$ Department of Rheumatology, University of Debrecen Faculty of Medicine,

Nagyerdei str 98, Debrecen 4032, Hungary

Full list of author information is available at the end of the article
}

(C) The Author(s). 2019 Open Access This article is distributed under the terms of the Creative Commons Attribution 4.0 International License (http://creativecommons.org/licenses/by/4.0/), which permits unrestricted use, distribution, and reproduction in any medium, provided you give appropriate credit to the original author(s) and the source, provide a link to the Creative Commons license, and indicate if changes were made. The Creative Commons Public Domain Dedication waiver (http://creativecommons.org/publicdomain/zero/1.0/) applies to the data made available in this article, unless otherwise stated. 


\section{Key messages}

- Rheumatoid arthritis is associated with impaired vascular pathophysiology

- Endothelial dysfunction and atherosclerosis may be associated with altered genetic signature

- Anti-TNF therapy may improve vascular function associated by changes in gene expression

\section{Introduction}

Accelerated atherosclerosis and increased cardiovascular (CV) morbidity and mortality have been associated with rheumatoid arthritis (RA). Vascular pathophysiology found in RA has been characterized by endothelial dysfunction, increasing arterial stiffness and overt atherosclerosis. These alterations are indicated by impaired brachial artery flow-mediated vasodilation (FMD), arterial pulse-wave velocity (PWV), and carotid plaque plus carotid intima-media thickness (IMT), respectively [1-4]. Several other non-invasive techniques have been available to detect vascular pathophysiology [1]. All these changes can be preclinically detected in RA with a negative history of CV disease (CVD) [2-6].

Targeted therapies including tumor necrosis factor $\alpha$ (TNF- $\alpha$ ) inhibitors are highly effective in RA $[7,8]$. The efficacy of these biologics may differ from patient to patient. Therefore, there is a high need for the identification of biomarkers including genetic signatures that may predict therapeutic response (R) vs non-response (NR) to biologics [9-12]. Indeed, gene expression profiling has been successfully used on tissue samples or blood for the identification of biomarkers and/or genome classifiers in various diseases [13, 14]. As described by several investigators, gene expression patterns of peripheral blood mononuclear cells (PBMCs) may be associated with response to therapies or disease progression [9-12, 15]. We have also performed gene expression studies and identified certain genomic signatures that may be associated with responses to infliximab [16] and tocilizumab [17]. Thus, we developed a standard protocol that could utilize genomics as biomarkers of disease or therapeutic outcomes $[16,17]$.

Both RA and atherosclerosis have strong genetic backgrounds. In RA, genome-wide association studies (GWAS) revealed numerous susceptibility alleles including HLADRB1, PTPN22, TRAF1/C5, STAT4, PADI4, IRF5, $F C G R$, IL2RA, IL2RB, CD40, CCR6, and CCL21. More than 40 single nucleotide polymorphisms (SNPs) have been associated with RA [18]. In atherosclerosis, a GWAS study carried out on more than 100,000 Europeans revealed association of more than 30 genes with coronary atherosclerosis. These included matrix molecule (e.g., ADAMTS7, $A N K S 1 A, C O L 4 A 1)$, lipid (e.g., $L P A, L D L R$ ), chemokine (e.g., CXCL12), and other genetic loci [19]. The roles of other "lipid-related genes" including $A B C A 1, A P O A 5$,
LCAT, CETP, and SORT1, as well as more than 50 "non-lipid" genes, have also been identified in atherosclerosis by GWAS [20]. HLADRB1 has been associated with both RA [18] and CVD [19].

It is also possible that RA and the associated CVD may have common genetic denominators. In this respect, mostly single-allele studies have been performed. Farragher et al. [21] reported that certain HLA-DRB1 alleles, mainly those functioning as shared epitope (SE), are associated with increased CV mortality in RA. For example, in comparison to RA patients carrying no or one SE allele, those with HLA-DRB1*01/*04 exert a three times higher risk for CV death [21]. Gonzalez-Gay et al. [22] also confirmed a relationship between SE alleles and CV morbidity and mortality. Studies on single non-HLA alleles were primarily performed by Gonzalez-Gay et al. [23, 24]. For example, the A1298C SNP in the MTHFR gene was associated with occurrence of $\mathrm{CV}$ events in RA patients. A SNP in the SMAD3 gene increased the risk for cerebrovascular accident in ACPA-negative RA patients. The HLA-DR1*04/*04 genotype and an SNP in the CD40 gene were associated with endothelial dysfunction and IMT, respectively. SNPs in the IRF5 and osteoprotegerin (OPG) genes could also be associated with vascular pathology. On the other hand, no associations between atherosclerosis/CVD or SNPs in the IFNG, JAK3, PON1, ADAMTS7, CARD8, CXCL12, ADIPOQ, and TLR4 alleles could be demonstrated [23, 24]. Yet, association of RA and atherosclerosis with complex genetic signatures has not yet been elucidated.

Therefore, we wished to determine associations between clinical responses to biologics, vascular pathophysiology, and gene expression patterns in a pilot study. Here, we performed global gene expression profiling in PBMCs of RA patients. We associated gene expression profiles (signatures) with (1) normal vs abnormal FMD, PWV, and IMT status of patients (study 1), (2) clinical response (cR) vs non-response (cNR) to 1-year biologic (etanercept, ETN, or certolizumab pegol, CZP) treatment as defined by EULAR response criteria (study 2), and (3) "vascular" response (vR) vs non-response (vNR) as defined by sufficient changes in FMD, PWV, and/or IMT upon 1-year ETN or CZP therapy (study 2).

\section{Materials and methods \\ Patients}

In study 1, 16 Caucasian, biologic-naive RA patients (15 females, 1 male), with a mean age of $53.7 \pm 5.7$ (range 42-60) years and a mean disease duration of $10.0 \pm 10.2$ (range 244 ), were included. In study 2, 19 biologic-naïve RA patients (18 females, 1 male; mean age $54.3 \pm 4.8$ [range 43-60] years, $12.1 \pm 10.9$ [range $2-44$ ] years) were recruited. Later, $12 \mathrm{pa}$ tients received ETN, and 7 CZP. All RA patients met the 2010 EULAR/ACR classification criteria for RA [25]. 
All blood samples were obtained after the subjects fasted overnight for $12 \mathrm{~h}$ locally between 8:00 AM and 9:00 AM before the first admission of biologics. Medication remained unchanged during the study.

The inclusion criteria in both studies included confirmed diagnosis of RA, age between 20 and 60, failure to respond to at least two DMARDs, active disease (DAS28 $>3.2$ ), and anti-TNF therapy-naïve patients. Corticosteroid therapy (prednisone $\leq 10 \mathrm{mg}$ per day) was allowed provided that the dosage had been stable for at least 2 months before entry. Also non-steroidal anti-inflammatory drugs (NSAID) were allowed in doses stable for at least 1 month before baseline. All patients received $10-25 \mathrm{mg} /$ week oral methotrexate (MTX) treatment, which had been stable for at least 4 weeks before baseline. Exclusion criteria included pregnancy or breastfeeding, current or recent malignancies, active infectious disease, patients with a history of arthritis or connective tissue disease other than RA, and smoking.

Disease activity was assessed by determining the 28-joint Disease Activity Score (DAS28) at baseline and then 12 months after the initiation of anti-TNF treatment in study 2 .

Clinical responder (cR vs cNR) status was determined after 12 months of treatment with either ETN or CZP by the EULAR response criteria originally described by Van Gestel et al. [26].

The Medical Research Council of Hungary gave ethical approval for this study (No. 9732-2/2012/EHR). In addition, the Institutional Review Board of the University of Debrecen Faculty of Medicine also approved the protocol. The study was in compliance with the Helsinki Declaration. Signed informed consent was obtained from all individuals providing blood samples.

\section{Assessment of vascular physiology by ultrasound}

Brachial artery FMD was assessed as described before [2, 27, 28]. In brief, ultrasound examination was performed on the right arm using a $10-\mathrm{MHz}$ linear array transducer (ultrasound system: HP Sonos 5500) by a single trained sonographer after $30 \mathrm{~min}$ of resting in a temperature-controlled room (basal value for FMD). A B-mode longitudinal section was obtained of the brachial artery above the antecubital fossa. In order to assess FMD, reactive hyperemia was induced by release of a pneumatic cuff around the forearm inflated to suprasystolic pressure for $4.5 \mathrm{~min}$. After deflation, the maximal flow velocity and the arterial diameter was continuously recorded for $90 \mathrm{~s}$. Flow velocities, the baseline diameter, and FMD were ECG gated and detected offline. FMD values were expressed as \% change from baseline (resting) value (FMD\%). In our previous work, we divided RA patients into "high (normal) FMD" and "low (impaired) FMD" subsets by defining a cutoff value of 5\% [2]. We used the same cutoff in the present studies.
The IMT measurements were carried out as described before [2, 28, 29]. Briefly, a duplex ultrasound system (HP Sonos 5500, $10 \mathrm{MHz}$ linear array transducer) was used to assess the common carotid arteries by a single observer. Longitudinal high-resolution B-mode ultrasound scans were employed over both the right and left common carotid arteries and were R-synchronized and recorded. The offline measurements were performed 1 $\mathrm{cm}$ proximal to the carotid bulb in the far wall. IMT was defined as the distance between the first and second echogenic lines from the lumen taking the average of 10 measurements on both sides. IMT values were expressed in millimeters. In our previous work, we divided RA patients into "high (increased) IMT" and "low (normal) IMT" subsets by using a cutoff value of $0.65 \mathrm{~mm}$ [2]. We used the same cutoff in the present studies.

With respect to arterial stiffness, PWV was calculated automatically by a TensioClinic arteriograph system (Tensiomed Ltd., Budapest, Hungary) with the quotient of the distance between the jugular fossa and symphysis as described before [28, 30, 31]. If an artery is elastic, PWV is low. With decreased arterial elasticity, PWV rises. The arteriograph assesses this parameter from the oscillometric data obtained from the $35 \mathrm{mmHg}$ suprasystolic pressure of the brachial artery [30, 31]. In order to obtain reproducible results, the patient had to rest in a supine position for at least $10 \mathrm{~min}$ before the assessment in a quiet room. PWV is expressed in meters per second. Based on our previous experience [28,31], we used a cutoff value of $8 \mathrm{~m} / \mathrm{s}$, where PWV $\leq 8 \mathrm{~m} / \mathrm{s}$ and PWV $>8 \mathrm{~m} / \mathrm{s}$ indicated "low (normal)" and "high (increased)" PWV, respectively.

In study 2 , we defined the arbitrary vascular responder status (vR vs vNR). Patients achieving at least $20 \%$ improvement in FMD, IMT, or PWV were considered vascular responders (vR). Patients achieving $\geq 20 \%$ improvement in at least two out of the three vascular parameters (FMD, IMT, PWV) were considered good vascular responders (GVR).

\section{PBMC and RNA isolation}

Venous peripheral blood samples were collected $(10 \mathrm{ml})$ in Venous Blood Vacuum Collection Tubes containing EDTA (BD Vacutainer K2EDTA). PBMCs were separated by Ficoll gradient centrifugation. Total RNA was extracted from PBMCs using Trizol reagent (Invitrogen), according to the manufacturer's protocol. RNA quality was checked on an Agilent Bioanalyzer 2100 (Agilent Technologies); all samples had a $28 \mathrm{~S} / 18 \mathrm{~S}$ ratio between 1.5 and 2.0, and the RNA Integrity Number was between 9 and 10. Quantity was determined by NanoDrop (Thermo Scientific).

\section{Microarray analysis and statistics}

Affymetrix GeneChip Human Primeview array was used to analyze global expression pattern of 28,869 
well-annotated genes. 3' IVT Expression Kit (Affymetrix) and GeneChip WT Terminal Labeling and Control Kit (Affymetrix) were used for amplifying and labeling 250 ng of RNA samples. Samples were hybridized at $45{ }^{\circ} \mathrm{C}$ for $16 \mathrm{~h}$, and then standard washing protocol was performed using a GeneChip Fluidics Station 450 and the arrays were scanned on a GeneChip Scanner 7G (Affymetrix).

Microarray data were analyzed by Genespring GX12 software (Agilent Technologies). Affymetrix data files were imported using the RMA algorithm and median normalization was performed. To identify differentially expressed genes between clinical conditions, statistical analysis was performed using a Mann-Whitney $U$ test; $p$ value $<0.05$ was considered to significant difference. We used hierarchical clustering and principal component analysis (PCA) to show the separation of clinical conditions by the differentially expressed genes. Microarray data were submitted to Gene Expression Omnibus (GEO), accession number: GSE126476.

Gene Ontology (GO) analysis was performed using Cytoscape 3.4.0 software (cytoscape.org) with the ClueGO application. The settings were the following: GO biological process, GO immune system process, and KEGG human diseases pathways; statistical options: two-sided hypergeometric test and Benjamini-Hochberg FDR for multiple testing correction. Significantly enriched GO categories were considered to $p$ value $<$ 0.05 and $\kappa$ score $<0.4$.
The association between clinical and various vascular responses was analyzed by Pearson's correlation $(p<0.05)$.

\section{Results}

Gene expression profiles may differentiate patients with normal vs impaired vascular pathophysiology

In study 1 , among the 16 RA patients, 11 had low ( $<5 \%$; red in Fig. 1a, d) and 5 had high ( $\geq 5 \%$; blue) FMD values (Fig. 1a). Similarly, low $(\leq 0.65 \mathrm{~mm}$; red in Fig. $1 \mathrm{~b}, \mathrm{e})$ and high $(>0.65$ $\mathrm{mm}$; blue) IMT values were observed in 11 and 5 patients, respectively (Fig. 1b, e). PWV was low ( $\leq 8 \mathrm{~m} / \mathrm{s}$; red in Fig. 1c, f) in 9 and high $(>8 \mathrm{~m} / \mathrm{s}$; blue) in 7 patients (Fig. 1c, f).

Two genes (CD74, ZNF718) were up- and 12 genes were significantly downregulated in patients with impaired (low) FMD compared to those with normal (high) FMD. The 12 downregulated genes are FOLR3, ADM, HP, DSC2, ANXA3, LILRA5, PLSCR1, AKAP12, VNN2, TCN1, HDC, and NFIL3 (Additional file 1: Table S1; Fig. 1d).

Altogether 62 up- and 129 downregulated genes were associated with increased (high) versus normal (low) IMT. The upregulated genes included GOS2, NRGN, ITGA2B, C3, FLNA, IRF5, ABCC3, CAPNS1, IL2RG, CCL4L1, ACTN1, HLAB, HLAC, TNFAIP3, and MYO1G. Among others, PPP1CB, HLADRB4, IFNGR1, LRRN3, CCR2, CD46, IFI44L, IFIT1, TLR10, CD164, IFIT2, SMAD4, and SGPP1 genes were downregulated (Additional file 1: Table S1; Fig. 1e).

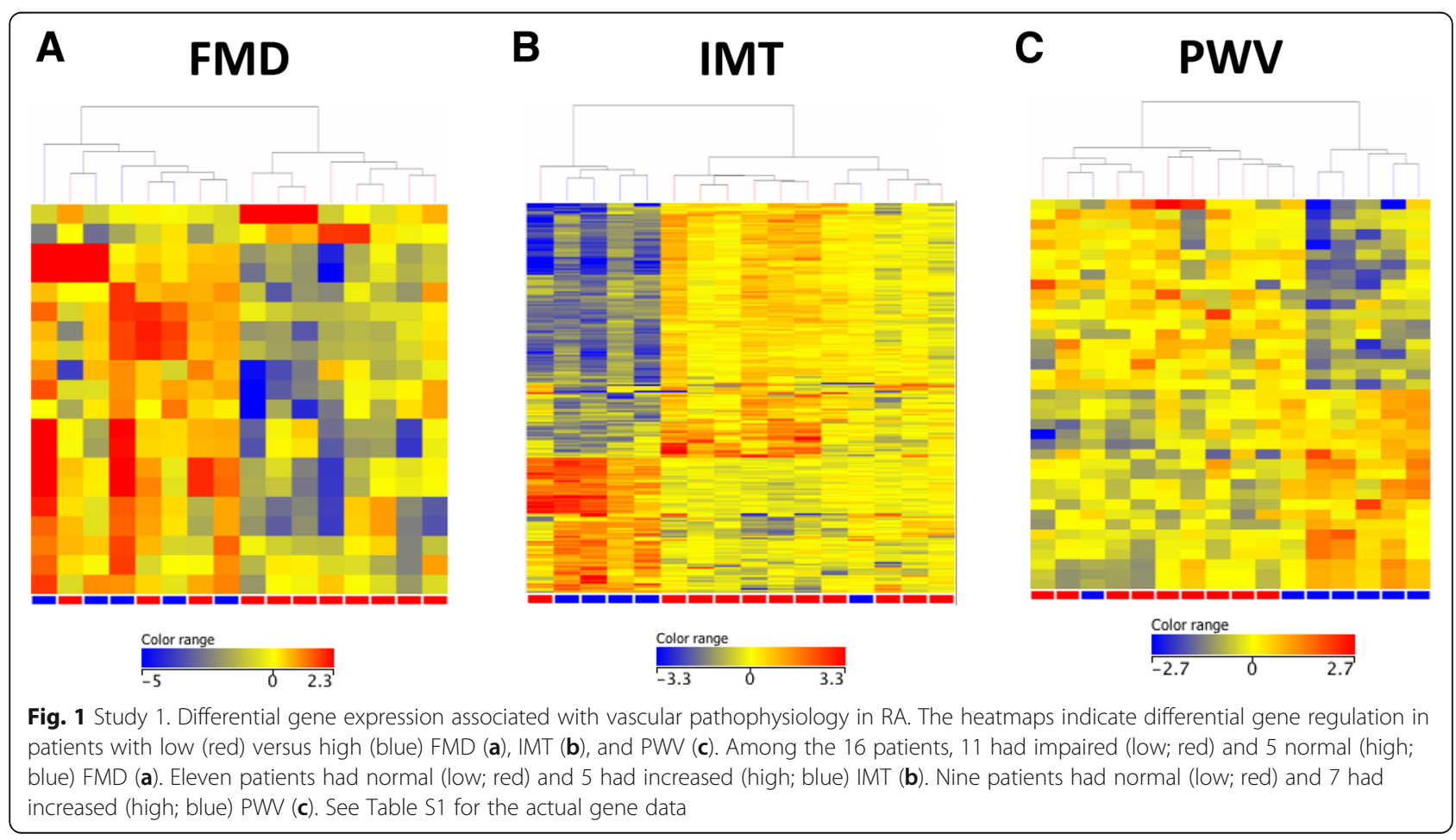


Finally, 32 genes showed differential expression between patients with increased (high) compared to normal (low) PWV. The changes were slight in most of these genes, and only two genes showed $\geq 2$-fold change difference between the high vs. low comparison, $H L A B / H L A C$ (upregulated) and LRRN3 (downregulated) (Additional file 1: Table S1; Fig. 1f).

\section{Association between clinical and vascular response upon 1-year anti-TNF therapy}

In study 2, 19 biologic-naïve RA patients were treated with either ETN or CZP for 12 months. Clinical (cR vs $\mathrm{cNR}$ ) and vascular (vR vs vNR) responder status was assessed after 1 year as described above. There were 13 cR and 6 cNR patients (Table 1; Fig. 2; cR: blue, cNR: red). According to the arbitrary definition of vascular responder status described above, 10 patients were FMD-20\% responders (Table 1; Fig. 3a), 9 were IMT-20\% responders (Table 1; Fig. 3b), and 8 were PWV-20\% responders (Table 1; Fig. 3c). Altogether, 8 patients achieved the GVR-20\% status (Table 1; Fig. 3d) (in the figures, responders are in blue and non-responders are in red). Out of the 19 patients, 5 patients achieved response in all three vascular parameters (FMD, IMT, PWV), and 3 in two parameters.
Clinical and vascular responder status of the treated patients was also compared (Table 2). When clinical responder status was correlated with FMD, IMT, or PWV responder status, as well as the GVR status, IMT response showed a tendency of correlation with the clinical response $(R=0.418, p=0.075)$ (Table 2). Yet, 8 FMD, 8 IMT, 6 PWV vRs, and 7 patients achieving GVR status were also cRs. On the other hand, 4, 5, 4, and 5 patients were both FMD, IMT, PWV, and global vascular NRs and cNRs, respectively (Table 2).

Differential baseline gene expression between clinical and vascular responders vs non-responders

First, baseline gene expression was associated with cR vs cNR status after 12 months of anti-TNF therapy (Fig. 2; Additional file 2: Table S2). Only 5 genes (HLADRB4, TMEM176A, TMEM176B, IFI44, and PLSCR1) were significantly ( $\geq 2$-fold) upregulated in $\mathrm{cR}$ vs $\mathrm{cNR}$ patients $(p<0.05)$. No significantly downregulated genes were found (Fig. 2; Additional file 2: Table S2).

Regarding vascular responses (vR) to biologics, the associations of baseline genetic signatures with vR vs vNR after 1-year ETN or CZP therapy were determined with respect to FMD, IMT, PWV, and GVR. FMD-20\% vR ( $\geq 20 \%$ improvement/increase in FMD after 12 months) vs vNR was

Table 1 Clinical and vascular responder status in ETN- or CZP-treated RA patients $(n=19)$ (study 2)

\begin{tabular}{|c|c|c|c|c|c|c|}
\hline Subject & Biologic & Clinical response & FMD response & IMT response & PWV response & Good vascular response \\
\hline 1 & ETN & $R$ & $R$ & $R$ & $R$ & $R$ \\
\hline 2 & ETN & $\mathrm{R}$ & $N R$ & $\mathrm{R}$ & NR & NR \\
\hline 3 & CZP & $\mathrm{R}$ & R & $\mathrm{R}$ & NR & $\mathrm{R}$ \\
\hline 4 & ETN & R & NR & NR & NR & NR \\
\hline 5 & ETN & R & $\mathrm{R}$ & $N R$ & NR & NR \\
\hline 6 & ETN & $R$ & $\mathrm{R}$ & NR & $\mathrm{R}$ & $\mathrm{R}$ \\
\hline 7 & CZP & $\mathrm{R}$ & NR & $\mathrm{R}$ & NR & NR \\
\hline 8 & ETN & $R$ & $\mathrm{R}$ & R & R & R \\
\hline 9 & CZP & $\mathrm{R}$ & $\mathrm{R}$ & $\mathrm{R}$ & $\mathrm{R}$ & $\mathrm{R}$ \\
\hline 10 & ETN & $\mathrm{R}$ & NR & $\mathrm{R}$ & $\mathrm{R}$ & $\mathrm{R}$ \\
\hline 11 & ETN & $\mathrm{R}$ & NR & $N R$ & $N R$ & $N R$ \\
\hline 12 & ETN & R & R & $\mathrm{R}$ & $\mathrm{R}$ & $\mathrm{R}$ \\
\hline 13 & ETN & R & $R$ & $N R$ & $N R$ & $N R$ \\
\hline 14 & CZP & $N R$ & $\mathrm{R}$ & $N R$ & $N R$ & $N R$ \\
\hline 15 & ETN & $N R$ & NR & NR & NR & NR \\
\hline 16 & CZP & $N R$ & $N R$ & $N R$ & $N R$ & NR \\
\hline 17 & CZP & $N R$ & $N R$ & $N R$ & $\mathrm{R}$ & NR \\
\hline 18 & CZP & $N R$ & $N R$ & $N R$ & $N R$ & $N R$ \\
\hline 19 & ETN & $N R$ & $\mathrm{R}$ & $\mathrm{R}$ & $\mathrm{R}$ & $\mathrm{R}$ \\
\hline$R(n)$ & & 13 & 10 & 9 & 8 & 8 \\
\hline$N R(n)$ & & 6 & 9 & 10 & 11 & 11 \\
\hline
\end{tabular}

$R$ responder, $N R$ non-responder, ETN etanercept, CZP certolizumab pegol 
Table 2 Association of clinical and vascular responses in ETN- or CZP-treated RA patients ( $\mathrm{n=19)}($ Study 2)

\begin{tabular}{|l|l|c|c|c|c|c|c|c|c|}
\hline \multicolumn{2}{|c|}{} & \multicolumn{2}{|c|}{ FMD response } & \multicolumn{2}{l|}{ IMT response } & \multicolumn{2}{l|}{ PWV response } & \multicolumn{2}{|c|}{$\begin{array}{c}\text { Good Vascular } \\
\text { response }\end{array}$} \\
\cline { 2 - 11 } & $\mathrm{R}$ & $\mathrm{NR}$ & $\mathrm{R}$ & $\mathrm{NR}$ & $\mathrm{R}$ & $\mathrm{NR}$ & $\mathrm{R}$ & $\mathrm{NR}$ \\
\hline $\begin{array}{l}\text { Clinical } \\
\text { Response }\end{array}$ & $\mathrm{R}$ & $\mathbf{8}$ & $\mathbf{5}$ & $\mathbf{8}$ & $\mathbf{5}$ & $\mathbf{6}$ & $\mathbf{7}$ & $\mathbf{7}$ & $\mathbf{6}$ \\
\cline { 2 - 10 } & $\mathrm{NR}$ & $\mathbf{2}$ & $\mathbf{4}$ & $\mathbf{1}$ & $\mathbf{5}$ & $\mathbf{2}$ & $\mathbf{4}$ & $\mathbf{1}$ & $\mathbf{5}$ \\
\hline
\end{tabular}

${ }^{*}$ Grey cells indicate R-R and NR-NR associations in clinical vs vascular responses

associated with significant ( $\geq 2$-fold) upregulation of a single gene (NEFL) and downregulation of two genes (JUN and GYPB) $(p<0.05)$ (Fig. 3a; Additional file 2: Table S2). IMT-20\% vR ( $\geq 20 \%$ improvement/decrease in IMT after 12 months) vs vNR was associated with upregulation of 18 (e.g., various immunoglobulin and HLA genes, TNFRSF17, CD74, FCRL5, CD79A, IFITM3) and downregulation of 12 genes (e.g., CXCL5, ITGB3, NEFL) (Fig. 3b, Additional file 2: Table S2). PWV-20\% vR ( $\geq 20 \%$ improvement/decrease in PWV after 12 months) vs vNR was associated with upregulation of three genes (IFNG, JUN, and $C C L 4 L 1 / L 2)$ and downregulation of five genes (HLAC, GNB4, NRG1, NEFL, and FKBP5) (Fig. 3c, Additional file 2: Table S2). Finally, a good vascular response (GVR-20\%) was defined as improvement by $\geq 20 \%$ in at least two vascular parameters (increase in FMD, decrease in IMT or PWV). In this respect, GVR-20\% vR vs vNR was associated with the upregulation of 11 genes (e.g., various immunoglobulin genes, SCN3A, CD79A, and FCRL5) and downregulation of two genes (NEFL and CES1/CES1P1) (Fig. 3d, Additional file 2: Table S2).

\section{Clustering and network analysis of differentially expressed genes}

Gene Ontology (GO) analysis was performed in order to demonstrate functional categories of differentially expressed genes, which showed at least twofold change up- or downregulation in study 2. As IMT has been associated with differential expression of numerous genes, we performed GO on IMT-, but not on FMD- or PWV-associated genes. Figure 4 shows the overrepresented functional categories of differentially expressed IMT-associated genes. The highest

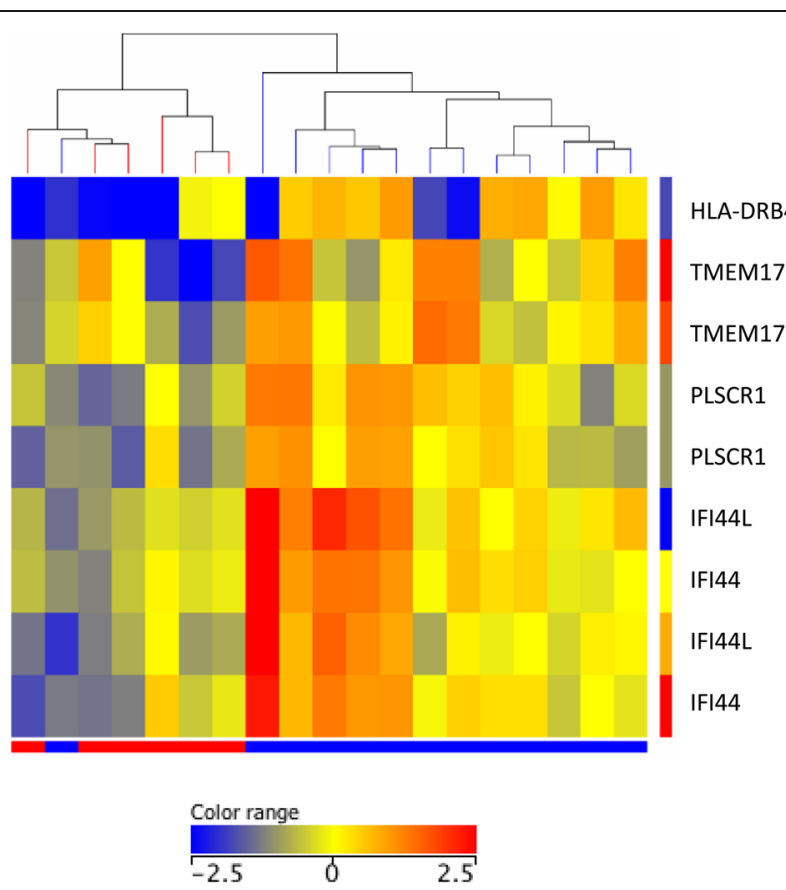

Fig. 2 Study 2. Differential gene expression associated with clinical response or non-response after 12 months of anti-TNF therapy. Altogether, 13 patients were responders and 6 were non-responders. Heatmap indicates differential gene regulation in clinical responders (blue) vs non-responders (red) 

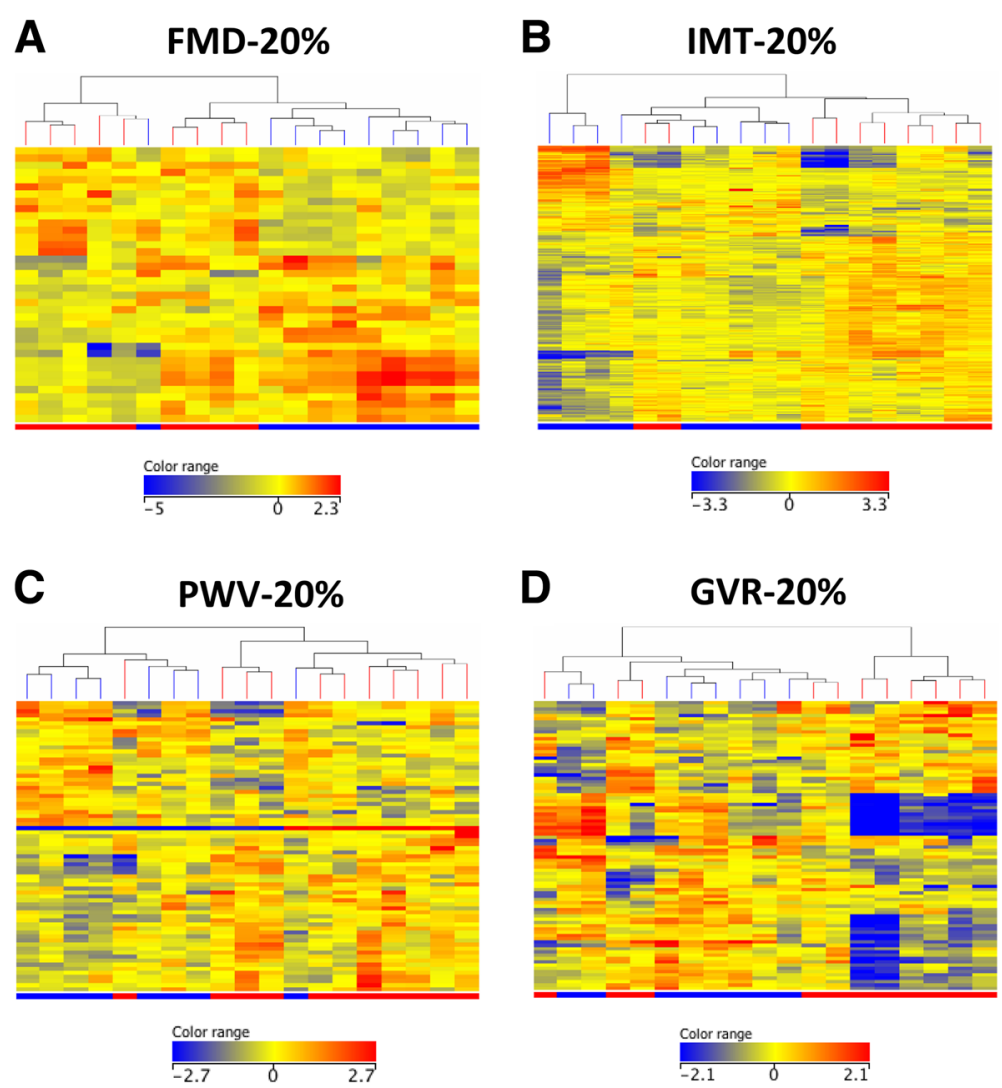

Fig. 3 Study 2. Differential gene expression associated with vascular responses to 12-month anti-TNF therapy. Among the 19 patients, 10 showed FMD-20\%, 9 demonstrated IMT-20\%, 8 showed PWV-20\%, and 8 exerted GVR-20\% responses. Heatmaps indicate differential gene regulation in FMD-20\% (a), IMT-20\% (b), PWV-20\% (c), and GVR-20\% (d) responders (blue) vs non-responders (red). See Table S2 for the actual gene data

number of genes showing significant enrichment was involved in positive regulation of immune effector process, regulation of glucose transport, Golgi vesicle budding, and others (Fig. 4).

\section{Discussion}

Accelerated atherosclerosis, as well as increased CV morbidity and mortality, has been associated with RA [2, 4, 32, 33]. Several HLA and non-HLA genes have been implicated in susceptibility to RA $[18,34,35]$ and atherosclerosis $[19,22,36]$. There may be common genetic factors underlying both RA and atherosclerosis [21, 23, 24]. Previous genetic studies mostly revealed the involvement of single SNPs in the background of RA-driven atherosclerosis. Thus, both HLA-DRB1 [21, 22, 37] and non-HLA alleles $[23,24]$ have been detected in this respect. To our knowledge, no complex genomic studies have been performed and published in association with CV pathophysiology or the effect of anti-TNF therapy on vascular function in RA. Therefore, we conducted a pilot study on a group of RA patients and compared clinical data, vascular pathophysiology, and patterns of differentially expressed genes.
Some genes were differentially regulated in association with FMD, IMT, and PWV or in conjunction to clinical and vascular responses to anti-TNF treatment. As discussed above, the Spanish group described numerous SNPs associated with RA-related CV disease [23, 24]. The genes identified by them, similarly to our study, encoded HLA, pro-inflammatory cytokines, and several other molecules related to autoimmunity and inflammation. As no complex genomic study or genetic study on biologic-treated patients have been conducted, our results cannot be compared to any previous studies.

In study 1, we applied pre-determined cutoff values for FMD, IMT, and PWV in order to define and to compare abnormal and normal vascular functions in 16 RA patients. $A \geq 2$-fold change in gene expression was considered meaningful and significant. Some genes were differentially expressed in RA patients with abnormal (high) vs normal (low) IMT. Much fewer genes were associated with abnormal (low) FMD, and only one gene was up- and one gene was downregulated in patients with abnormal (high) PWV. Differentially expressed genes primarily included MHC-related genes, but also genes encoding cytokine, adhesion molecule, integrin, and interferon-related ones. 


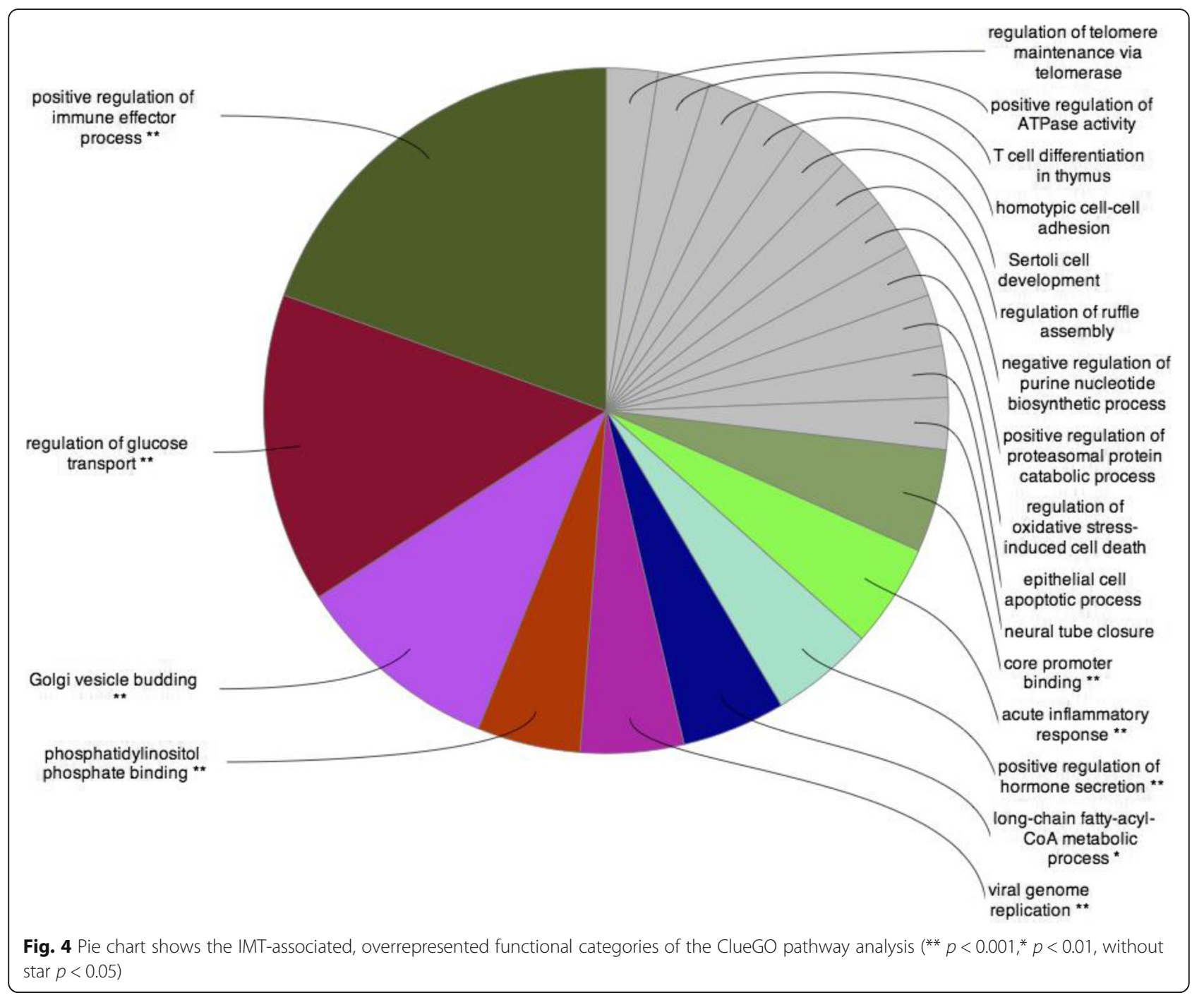

In study 2, we treated 19 RA patients with TNF inhibitors (either ETN or CZP) for 1 year. In the end, 13 patients showed a clinical response to treatment. We defined vascular responses as at least $20 \%$ improvement in FMD, IMT, and PWV after 12 months of biologic treatment. We also defined GVR as $\geq 20 \%$ improvement in at least two vascular parameters. Only 10-13 out of 19 patients had concordance between clinical and any vascular response. Clinical response was associated with the upregulation of five genes only. Again, some genes were differentially expressed in IMT responder RA patients compared to non-responders. Very few genes were associated with FMD, PWV, or GVR responses to anti-TNF therapy. Here, differentially expressed genes primarily included immunoglobulin- and HLA-related genes, but also other genes including cytokine, chemokine, and interferon-related genes.

A few particular genes were picked up in multiple analyses. Just to present a few interesting examples, two vanin genes (VNN1 and VNN2) showed differential expression in study 1 . Several interferon-related genes (e.g., IFI44, IFIT1, IFITM3, IRF5) exerted differential expression in both studies. In SNP studies, IRF5 genetic variants were associated with $\mathrm{CV}$ disease in $\mathrm{RA}$ [38]. Although the role of vanins in RA is unknown, these molecules have been implicated in fibrosis and vascular pathology underlying other autoimmune conditions [39] and atherosclerosis [40]. The gene for neurofilament light polypeptide (NEFL) was significantly associated with FMD, IMT, PWV, and GVR responses in study 2. Neurofilament proteins have been associated with nervous system damage in SLE [41]. However, cytoskeletal neurofilaments have also been detected in the RA synovium [42]. Antibodies to neurofilament proteins may play a role in RA, as well as SLE [43]. Leucine-rich repeat neuronal 3 (LRRN3) gene exerted differential expression in RA patients with abnormal IMT and PWV in study 1 . This gene has been associated with aging [44]. 
In general, leucine-rich repeat kinases (LRRK) are involved in neuroinflammation, but also RA and other arthritides [45]. Finally, in study 2, JUN, the gene encoding the jun proto-oncogene, also showed differential expression in association with FMD-20\% and PWV-20\% responses. This molecule [46, 47], as well as c-Jun N-terminal kinase (JNK) $[48,49]$, has long been associated with the pathogenesis of RA and atherosclerosis. Certainly, several other genes and their roles in RA and/ or $\mathrm{CV}$ disease could have been demonstrated.

\section{Conclusion}

In order to demonstrate the function of the genes showing differential expression in association with RA-related vascular pathophysiology, GO analysis was performed on IMT-associated, overrepresented genes. In general, the differential expression of numerous genes was associated with FMD, IMT, and PWV in RA. Genetic signatures were also associated with clinical and vascular responses to anti-TNF therapy. The major strength of this study is that after SNP studies, this may be the first one studying more complex genetic signatures in relation to RA-associated atherosclerosis. Our study may have limitations including the relatively low number of patients and that multiple testing could not be carried out, so this study may lack power. Therefore, after this pilot study, further, larger ones need to be conducted in order to further delineate the genetic/genomic background of RA-related vascular disease.

\section{Additional files}

Additional file 1: Table S1. Association of gene expression profiles with vascular pathophysiology in biologic-naive RA patients $(n=16)$ (study 1). (DOCX $35 \mathrm{~kb})$

Additional file 2: Table S2. Association of gene expression profiles with clinical and vascular responses upon anti-TNF therapy in RA patients $(n=19)$ (study 2). (DOCX $22 \mathrm{~kb}$ )

\begin{abstract}
Abbreviations
ACPA: Anti-citrullinated peptide antibody; ACR: American College of Rheumatology; cNR: Clinical non-response/responder; CR: Clinical response/ responder; CV: Cardiovascular; CVD: Cardiovascular disease; CZP: Certolizumab pegol; DAS28: 28-Joint disease activity scale; DMARD: Disease-modifying antirheumatic drug; ETN: Etanercept; EULAR: European League Against Rheumatism; FMD: Flow-mediated vasodilation; GO: Gene Ontology; GVR: Good vascular response/responder; GWAS: Genome-wide association study; HLA: Human leukocyte antigen; IMT: Intima-media thickness; MTX: Methotrexate; NR: Non-response; NSAID: Non-steroidal anti-inflammatory drug; PBMC: Peripheral blood mononuclear cell; PWV: Pulse-wave velocity; R: Responder; RA: Rheumatoid arthritis; SE: Shared epitope; SLE: Systemic lupus erythematosus; SNP: Single nucleotide polymorphism; TNF-a: Tumor necrosis factor alpha; vNR: Vascular nonresponse/responder; vR: Vascular response/responder
\end{abstract}

\section{Acknowledgements}

This work was supported by an OTKA K10073 grant (H.P.B and Z.S.) donated by the Hungarian Scientific Research Fund; by the European Union and the State of Hungary co-financed by the European Social Fund in the framework of TAMOP-4.2.4.A/2-11/1-2012-0001 'National Excellence Program'(Z.S.); also by the European Union grants GINOP-2.3.2-15-2016-00015 and GINOP-2.3.215-2016-00050 (Z.S.)

\section{Funding}

This work was supported by an OTKA K10073 grant (H.P.B and Z.S.) donated by the Hungarian Scientific Research Fund; by the European Union and the State of Hungary co-financed by the European Social Fund in the framework of TAMOP-4.2.4.A/2-11/1-2012-0001 'National Excellence Program'(Z.S.); also by the European Union grants GINOP-2.3.2-15-2016-00015 and GINOP-2.3.215-2016-00050 (Z.S.).

\section{Availability of data and materials}

N/A.

\section{Authors' contributions}

SP contributed to the study design, gene expression analysis, statistics, and manuscript draft. TB contributed to the study design, patient examination, and manuscript draft. EV contributed to the patient examination, patient data, and performance of ultrasound analysis. AH contributed to the patient examination, patient data, and data collection and analysis. AP contributed to the patient data, data collection and analysis, and biobanking. AV contributed to the patient examination and patient data. NB contributed to the patient examination, patient data, and performance of ultrasound analysis. SSzam contributed to the patient examination and patient data. MC contributed to the gene expression analysis. GK contributed to the ultrasound analysis. ZSzab contributed to the patient examination and patient data. ZN contributed to the study design, data analysis, and statistics. GS contributed to the patient examination and patient data and gave general advice. SSzán contributed to the patient examination and patient data and gave general advice. GZ contributed to the gene expression analysis and data analysis. LN contributed to the gene expression analysis and data analysis and gave general advice. ZSzek is the project manager, gave expert advices, and contributed to the study design and manuscript draft. All authors read and approved the final manuscript.

\section{Authors' information}

See above, cover page.

\section{Ethics approval and consent to participate}

The Medical Research Council of Hungary gave ethical approval for this study (No. 9732-2/2012/EHR). In addition, the Institutional Review Board of the University of Debrecen Faculty of Medicine also approved the protocol. The study that was in compliance with the Helsinki Declaration. Ethical approval (No. 1046-63/2015) was obtained from the Regional/Institutional Review Board of Miskolc University. All patients signed informed consent. The study was performed according to the Declaration of Helsinki.

\section{Consent for publication}

No data that could identify single patients are presented therefore this consent is not needed.

\section{Competing interests}

The authors declare that they have no competing interests.

\section{Publisher's Note}

Springer Nature remains neutral with regard to jurisdictional claims in published maps and institutional affiliations.

\section{Author details}

${ }^{1}$ Department of Biochemistry and Molecular Biology, University of Debrecen Faculty of Medicine, Debrecen, Hungary. '2Department of Rheumatology, University of Debrecen Faculty of Medicine, Nagyerdei str 98, Debrecen 4032 Hungary. ${ }^{3}$ Department of Internal Medicine, University of Debrecen Faculty of Medicine, Debrecen, Hungary. ${ }^{4}$ Department of Angiology, University of Debrecen Faculty of Medicine, Debrecen, Hungary. ${ }^{5}$ Department of Sports Medicine, University of Debrecen Faculty of Medicine, Debrecen, Hungary. ${ }^{6}$ UD Genomed Ltd., Debrecen, Hungary. 
Received: 16 October 2018 Accepted: 8 March 2019 Published online: 15 April 2019

\section{References}

1. Kerekes G, Soltesz P, Nurmohamed MT, Gonzalez-Gay MA, Turiel M, Vegh E, Shoenfeld Y, Mclnnes I, Szekanecz Z. Validated methods for assessment of subclinical atherosclerosis in rheumatology. Nat Rev Rheumatol. 2012;8(4): 224-34.

2. Kerekes G, Szekanecz Z, Der H, Sandor Z, Lakos G, Muszbek L, Csipo I, Sipka S, Seres I, Paragh G, et al. Endothelial dysfunction and atherosclerosis in rheumatoid arthritis: a multiparametric analysis using imaging techniques and laboratory markers of inflammation and autoimmunity. J Rheumatol. 2008;35(3):398-406.

3. Peters MJ, van Eijk IC, Smulders YM, Serne E, Dijkmans BA, van der HorstBruinsma IE, Nurmohamed MT. Signs of accelerated preclinical atherosclerosis in patients with ankylosing spondylitis. J Rheumatol. 2010;37(1):161-6.

4. Gonzalez-Gay MA, Gonzalez-Juanatey C, Vazquez-Rodriguez TR, Martin J, Llorca J. Endothelial dysfunction, carotid intima-media thickness, and accelerated atherosclerosis in rheumatoid arthritis. Semin Arthritis Rheum. 2008;38(2):67-70.

5. Bodnar N, Kerekes G, Seres I, Paragh G, Kappelmayer J, Nemethne ZG, Szegedi G, Shoenfeld Y, Sipka S, Soltesz P, et al. Assessment of subclinical vascular disease associated with ankylosing spondylitis. J Rheumatol. 2011;38(4):723-9.

6. Gonzalez-Juanatey C, Vazquez-Rodriguez TR, Miranda-Filloy JA, Dierssen T, Vaqueiro I, Blanco R, Martin J, Llorca J, Gonzalez-Gay MA. The high prevalence of subclinical atherosclerosis in patients with ankylosing spondylitis without clinically evident cardiovascular disease. Medicine (Baltimore). 2009;88(6):358-65.

7. Karampetsou MP, Liossis SN, Sfikakis PP. TNF-alpha antagonists beyond approved indications: stories of success and prospects for the future. QJM. 2010;103(12):917-28

8. Smolen JS, Aletaha D, Koeller M, Weisman MH, Emery P. New therapies for treatment of rheumatoid arthritis. Lancet. 2007;370(9602):1861-74

9. Verweij CL. Pharmacogenetics: anti-TNF therapy in RA--towards personalized medicine? Nat Rev Rheumatol. 2011;7(3):136-8.

10. Szekanecz Z, Mesko B, Poliska S, Vancsa A, Szamosi S, Vegh E, Simkovics E, Laki J, Kurko J, Besenyei T, et al. Pharmacogenetics and pharmacogenomics in rheumatology. Immunol Res. 2013;56(2-3):325-33.

11. Cronstein BN. Pharmacogenetics in the rheumatic diseases, from pret-aporter to haute couture. Nat Clin Pract Rheumatol. 2006;2(1):2-3.

12. Davila L, Ranganathan P. Pharmacogenetics: implications for therapy in rheumatic diseases. Nat Rev Rheumatol. 2011;7(9):537-50.

13. Mesko B, Poliska S, Szegedi A, Szekanecz Z, Palatka K, Papp M, Nagy L. Peripheral blood gene expression patterns discriminate among chronic inflammatory diseases and healthy controls and identify novel targets. BMC Med Genet. 2010;3:15.

14. Mesko B, Poliska S, Nagy L. Gene expression profiles in peripheral blood for the diagnosis of autoimmune diseases. Trends Mol Med. 2011;17(4): 223-33.

15. Aziz H, Zaas A, Ginsburg GS. Peripheral blood gene expression profiling for cardiovascular disease assessment. Genomic Med. 2007;1(3-4):105-12.

16. Mesko B, Poliska S, Vancsa A, Szekanecz Z, Palatka K, Hollo Z, Horvath A, Steiner L, Zahuczky G, Podani J, et al. Peripheral blood derived gene panels predict response to infliximab in rheumatoid arthritis and Crohn's disease. Genome Med. 2013;5(6):59.

17. Mesko B, Poliska S, Szamosi S, Szekanecz Z, Podani J, Varadi C, Guttman A, Nagy L. Peripheral blood gene expression and lgG glycosylation profiles as markers of tocilizumab treatment in rheumatoid arthritis. J Rheumatol. 2012; 39(5):916-28.

18. Kurko J, Besenyei T, Laki J, Glant T, Mikecz K, Szekanecz Z. Genetics of rheumatoid arthritis - a comprehensive review. Clin Rev Allergy Immunol. 2013;45(2):170-9.

19. Lusis AJ. Genetics of atherosclerosis. Trends Genet. 2012;28(6):267-75.

20. Marian AJ. The enigma of genetics etiology of atherosclerosis in the postGWAS era. Curr Atheroscler Rep. 2012;14(4):295-9.

21. Farragher TM, Goodson NJ, Naseem H, Silman AJ, Thomson W, Symmons D, Barton A. Association of the HLA-DRB1 gene with premature death, particularly from cardiovascular disease, in patients with rheumatoid arthritis and inflammatory polyarthritis. Arthritis Rheum. 2008;58(2):359-69.

22. Gonzalez-Gay MA, Gonzalez-Juanatey C, Lopez-Diaz MJ, Pineiro A, GarciaPorrua C, Miranda-Filloy JA, Ollier WE, Martin J, Llorca J. HLA-DRB1 and persistent chronic inflammation contribute to cardiovascular events and cardiovascular mortality in patients with rheumatoid arthritis. Arthritis Rheum. 2007;57(1):125-32.

23. Remuzgo-Martinez S, Genre F, Lopez-Mejias R, Ubilla B, Mijares V, Pina T, Corrales A, Blanco R, Martin J, Llorca J, et al. Expression of osteoprotegerin and its ligands, RANKL and TRAIL, in rheumatoid arthritis. Sci Rep. 2016;6: 29713.

24. Lopez-Mejias R, Castaneda S, Gonzalez-Juanatey C, Corrales A, Ferraz-Amaro I, Genre F, Remuzgo-Martinez S, Rodriguez-Rodriguez L, Blanco R, Llorca J, et al. Cardiovascular risk assessment in patients with rheumatoid arthritis: the relevance of clinical, genetic and serological markers. Autoimmun Rev. 2016; 15(11):1013-30.

25. Aletaha D, Neogi T, Silman AJ, Funovits J, Felson DT, Bingham CO 3rd, Birnbaum NS, Burmester GR, Bykerk VP, Cohen MD, et al. 2010 rheumatoid arthritis classification criteria: an American College of Rheumatology/ European league against rheumatism collaborative initiative. Arthritis Rheum. 2010;62(9):2569-81.

26. van Gestel AM, Prevoo ML, van 't Hof MA, van Rijswijk MH, van de Putte LB, van Riel PL. Development and validation of the European League Against Rheumatism response criteria for rheumatoid arthritis. Comparison with the preliminary American College of Rheumatology and the World Health Organization/International League Against Rheumatism Criteria. Arthritis Rheum. 1996:39(1):34-40.

27. Corretti MC, Anderson TJ, Benjamin EJ, Celermajer D, Charbonneau F, Creager MA, Deanfield J, Drexler H, Gerhard-Herman M, Herrington D, et al. Guidelines for the ultrasound assessment of endothelial-dependent flowmediated vasodilation of the brachial artery: a report of the International Brachial Artery Reactivity Task Force. J Am Coll Cardiol. 2002;39(2):257-65.

28. Soltesz P, Der H, Kerekes G, Szodoray P, Szucs G, Danko K, Shoenfeld Y, Szegedi G, Szekanecz Z. A comparative study of arterial stiffness, flowmediated vasodilation of the brachial artery, and the thickness of the carotid artery intima-media in patients with systemic autoimmune diseases. Clin Rheumatol. 2009;28(6):655-62.

29. Kanters SD, Algra A, van Leeuwen MS, Banga JD. Reproducibility of in vivo carotid intima-media thickness measurements: a review. Stroke. 1997;28(3):665-71.

30. Baulmann J, Schillings U, Rickert S, Uen S, Dusing R, Illyes M, Cziraki A Nickering G, Mengden T. A new oscillometric method for assessment of arterial stiffness: comparison with tonometric and piezo-electronic methods. J Hypertens. 2008;26(3):523-8.

31. Timar O, Soltesz P, Szamosi S, Der H, Szanto S, Szekanecz Z, Szucs G. Increased arterial stiffness as the marker of vascular involvement in systemic sclerosis. J Rheumatol. 2008:35(7):1329-33.

32. Shoenfeld Y, Gerli R, Doria A, Matsuura E, Cerinic MM, Ronda N, Jara L, Abu-Shakra M, Meroni PL, Sherer Y. Accelerated atherosclerosis in autoimmune rheumatic diseases. Circulation. 2005;112(21):3337-47.

33. Agca R, Heslinga SC, Rollefstad S, Heslinga M, Mclnnes IB, Peters MJ, Kvien TK, Dougados M, Radner H, Atzeni F, et al. EULAR recommendations for cardiovascular disease risk management in patients with rheumatoid arthritis and other forms of inflammatory joint disorders: 2015/2016 update. Ann Rheum Dis. 2017;76(1):17-28.

34. de Vries R. Genetics of rheumatoid arthritis: time for a change! Curr Opin Rheumatol. 2011;23(3):227-32.

35. Gregersen PK, Silver J, Winchester RJ. The shared epitope hypothesis. An approach to understanding the molecular genetics of susceptibility to rheumatoid arthritis. Arthritis Rheum. 1987;30(11):1205-13.

36. Schunkert H, Konig IR, Kathiresan S, Reilly MP, Assimes TL, Holm H, Preuss M, Stewart AF, Barbalic M, Gieger C, et al. Large-scale association analysis identifies 13 new susceptibility loci for coronary artery disease. Nat Genet. 2011;43(4):333-8.

37. Gonzalez-Gay MA, Gonzalez-Juanatey C, Llorca J, Ollier WE, Martin J. Contribution of HLA-DRB1 shared epitope alleles and chronic inflammation to the increased incidence of cardiovascular disease in rheumatoid arthritis: comment on the article by Farragher et al. Arthritis Rheum. 2008;58(8):2584 author reply 2584-2585.

38. Garcia-Bermudez M, Lopez-Mejias R, Genre F, Castaneda S, Llorca J, Gonzalez-Juanatey C, Corrales A, Ubilla B, Miranda-Filloy JA, Pina T, et al. Interferon regulatory factor 5 genetic variants are associated with cardiovascular disease in patients with rheumatoid arthritis. Arthritis Res Ther. 2014;16(4):R146.

39. van Diepen JA, Jansen PA, Ballak DB, Hijmans A, Rutjes FP, Tack CJ, Netea MG, Schalkwijk J, Stienstra R. Genetic and pharmacological inhibition of vanin-1 activity in animal models of type 2 diabetes. Sci Rep. 2016;6:21906. 
40. Hu YW, Wu SG, Zhao JJ, Ma X, Lu JB, Xiu JC, Zhang Y, Huang C, Qiu YR, Sha YH, et al. VNN1 promotes atherosclerosis progression in apoE-/- mice fed a highfat/high-cholesterol diet. J Lipid Res. 2016;57(8):1398-411.

41. Trysberg E, Nylen K, Rosengren LE, Tarkowski A. Neuronal and astrocytic damage in systemic lupus erythematosus patients with central nervous system involvement. Arthritis Rheum. 2003;48(10):2881-7.

42. Gronblad M, Konttinen YT, Korkala O, Liesi P, Hukkanen M, Polak JM. Neuropeptides in synovium of patients with rheumatoid arthritis and osteoarthritis. J Rheumatol. 1988;15(12):1807-10.

43. Salih AM, Nixon NB, Dawes PT, Mattey DL. Prevalence of antibodies to neurofilament polypeptides in patients with rheumatoid arthritis complicated by peripheral neuropathy. Clin Exp Rheumatol. 1998;16(6):689-94.

44. Kochunov P, Charlesworth J, Winkler A, Hong LE, Nichols TE, Curran JE, Sprooten E, Jahanshad N, Thompson PM, Johnson MP, et al. Transcriptomics of cortical gray matter thickness decline during normal aging. Neuroimage. 2013:82:273-83

45. Li N, Ma J, Li K, Guo C, Ming L. Different contributions of CDKAL1, KIF21B, and LRRK2/MUC19 polymorphisms to SAPHO syndrome, rheumatoid arthritis, ankylosing spondylitis, and seronegative spondyloarthropathy Genet Test Mol Biomarkers. 2017;21(2):122-6.

46. Dooley S, Herlitzka I, Hanselmann R, Ermis A, Henn W, Remberger K, Hopf T, Welter C. Constitutive expression of c-fos and c-jun, overexpression of ets-2, and reduced expression of metastasis suppressor gene $\mathrm{nm} 23-\mathrm{H} 1$ in rheumatoid arthritis. Ann Rheum Dis. 1996:55(5):298-304.

47. Sozen E, Karademir B, Yazgan B, Bozaykut P, Ozer NK. Potential role of proteasome on $\mathrm{c}$-jun related signaling in hypercholesterolemia induced atherosclerosis. Redox Biol. 2014;2:732-8.

48. Han Z, Boyle DL, Aupperle KR, Bennett B, Manning AM, Firestein GS. Jun Nterminal kinase in rheumatoid arthritis. J Pharmacol Exp Ther. 1999;291(1): 124-30.

49. Kwok KHM, Cheng KKY, Hoo RLC, Ye D, Xu A, Lam KSL. Adipose-specific inactivation of JNK alleviates atherosclerosis in apoE-deficient mice. Clin Sci (Lond). 2016:130(22):2087-100.

Ready to submit your research? Choose BMC and benefit from:

- fast, convenient online submission

- thorough peer review by experienced researchers in your field

- rapid publication on acceptance

- support for research data, including large and complex data types

- gold Open Access which fosters wider collaboration and increased citations

- maximum visibility for your research: over $100 \mathrm{M}$ website views per year

At $\mathrm{BMC}$, research is always in progress.

Learn more biomedcentral.com/submissions 